\title{
Asymmetrical distribution of non-conserved regulatory sequences at PHOX2B is reflected at the ENCODE loci and illuminates a possible genome-wide trend
}

\author{
David M McGaughey ${ }^{\dagger}$, Zachary E Stine ${ }^{\dagger 1}$, Jimmy L Huynh ${ }^{1}$, Ryan M Vinton ${ }^{1}$ \\ and Andrew S McCallion*1,2
}

Address: ${ }^{1}$ McKusick - Nathans Institute of Genetic Medicine, Johns Hopkins University School of Medicine, 733 N. Broadway, BRB Suite 449 , Baltimore, MD 21205, USA and 2Department of Molecular and Comparative Pathobiology, Johns Hopkins University School of Medicine, Baltimore, MD 21205, USA

Email: David M McGaughey - davidm@jhmi.edu; Zachary E Stine - zstine1@jhmi.edu; Jimmy L Huynh - jimh@jhmi.edu; Ryan M Vinton - rvinton1@jhmi.edu; Andrew S McCallion* - andy@jhmi.edu

* Corresponding author †Equal contributors

Published: 7 January 2009

BMC Genomics 2009, 10:8 doi:10.1 186/147|-2164-10-8
Received: 8 July 2008

Accepted: 7 January 2009

This article is available from: http://www.biomedcentral.com/I47/-2/64/I0/8

(c) 2009 McGaughey et al; licensee BioMed Central Ltd.

This is an Open Access article distributed under the terms of the Creative Commons Attribution License (http://creativecommons.org/licenses/by/2.0), which permits unrestricted use, distribution, and reproduction in any medium, provided the original work is properly cited.

\begin{abstract}
Background: Transcriptional regulatory elements are central to development and interspecific phenotypic variation. Current regulatory element prediction tools rely heavily upon conservation for prediction of putative elements. Recent in vitro observations from the ENCODE project combined with in vivo analyses at the zebrafish phox $2 b$ locus suggests that a significant fraction of regulatory elements may fall below commonly applied metrics of conservation. We propose to explore these observations in vivo at the human $P H O X 2 B$ locus, and also evaluate the potential evidence for genome-wide applicability of these observations through a novel analysis of extant data.

Results: Transposon-based transgenic analysis utilizing a tiling path proximal to human $P H O X 2 B$ in zebrafish recapitulates the observations at the zebrafish phox $2 b$ locus of both conserved and nonconserved regulatory elements. Analysis of human sequences conserved with previously identified zebrafish phox $2 b$ regulatory elements demonstrates that the orthologous sequences exhibit overlapping regulatory control. Additionally, analysis of non-conserved sequences scattered over $135 \mathrm{~kb} 5^{\prime}$ to PHOX2B, provides evidence of non-conserved regulatory elements positively biased with close proximity to the gene. Furthermore, we provide a novel analysis of data from the ENCODE project, finding a nonuniform distribution of regulatory elements consistent with our in vivo observations at PHOX2B. These observations remain largely unchanged when one accounts for the sequence repeat content of the assayed intervals, when the intervals are sub-classified by biological role (developmental versus nondevelopmental), or by gene density (gene desert versus non-gene desert).

Conclusion: While regulatory elements frequently display evidence of evolutionary conservation, a fraction appears to be undetected by current metrics of conservation. In vivo observations at the PHOX2B locus, supported by our analyses of in vitro data from the ENCODE project, suggest that the risk of excluding non-conserved sequences in a search for regulatory elements may decrease as distance from the gene increases. Our data combined with the ENCODE data suggests that this may represent a genome wide trend.
\end{abstract}




\section{Background}

Transcriptional regulatory elements play a critical role in disease [1-3], development [4,5], and interspecific phenotypic variation [6-8]. However, unlike coding sequences, there is no universal vocabulary that can infer their biological relevance based on sequence composition alone, and, unlike structural RNAs, there is no associated secondary structure which can aid in their identification.

Comparative genomic sequence analysis has proven to be a powerful tool for the identification of putatively functional sequences, predicting functional significance based on evolutionary conservation [9-15]. A logical extrapolation of this approach has been to focus on highly or ultraconserved non-coding elements as regions presumably enriched for regulatory sequences [9,16-18]. However, functional regulatory elements have also been identified using less stringent definitions of constraint [11,19-22]. Taken together these studies suggest that no single evolutionary distance can capture all functional regulatory elements. Consistent with this are reports of reduced promoter evolutionary constraint between human and chimpanzee [23], wide spread cis-regulatory element shuffling [24] and mammalian putative regulatory regions that do not align to other mammalian genomes [25-28]. Furthermore, we recently completed a comprehensive functional screen for regulatory activity by tiling sequences across the zebrafish phox $2 b$ locus and demonstrated that, at least at that locus, a significant fraction of regulatory sequences displayed no evidence of functional constraint [29]. However zebrafish and fugu, its most closely related sequenced genome, are substantially more evolutionarily separated than humans are from rodents (3-400 million years compared to 80-100 million years). Thus, one explanation for this observation is that functional non-conserved sequences are detectably conserved over shorter evolutionary distances. We directly address this idea by assaying sequences from a tiled interval of the human PHOX2B locus. We hypothesize that functional non-conserved sequences are likely present at the human locus in comparable frequency to the zebrafish phox $2 b$ ortholog [29]. PHOX2B is a three exon gene that spans approximately $4.9 \mathrm{~kb}$ and encodes a paired homeobox domain transcription factor. This developmentally critical gene is tightly regulated and mutations in its coding sequence have been implicated in several human pathologies, including central congenital hypoventilation syndrome, neuroblastoma, and Hirschsprung disease [3034].

The recent public release of data from the ENCODE project [25] provided a large data set to explore putative regulatory element conservation and distribution. Using protein occupancy and chromatin modification data from the ENCODE project, 1,394 of the most likely transcrip- tional regulatory elements were identified and termed putative transcriptional regulatory regions (pTRRs) [26]. Consistent with our observations at phox $2 b$, the ENCODE consortium reports a markedly low coincidence between pTRRs and multi-species constrained sequences $[25,26,35,36]$. While the enrichment of putative regulatory elements proximal to coding regions [35] has similarly been noted, the relative distribution of nonconserved proximal and distal intergenic pTRRs has not to our knowledge been examined thoroughly.

We set out to address three main questions using a transposon-based transgenic approach in zebrafish embryos. First, we tested whether the human PHOX2B locus contained functional non-conserved regulatory sequences in addition to conserved PHOX2B functional elements, similar to its zebrafish ortholog. We assayed the regulatory potential of a tiled interval encompassing ten kilobases (kb) upstream of $P H O X 2 B$ as well as the intronic sequences. Second, we assayed human PHOX2B non-coding sequences across a $135 \mathrm{~kb}$ interval that were conserved with previously identified zebrafish phox $2 b$ regulatory sequences, demonstrating that most overlap in their regulatory control; the functionality of these human to zebrafish orthologous sequences remains generally unchanged with increasing distance from the gene. Third, by using these regulatory sequences as "anchors," we tested flanking sequences that were not conserved and provide preliminary evidence of a trend that non-conserved regulatory sequences tend to lie more frequently proximal to the PHOX $2 B /$ phox $2 b$ exons. To test if this apparent non-uniform distribution of non-conserved regulatory elements represents a genome-wide characteristic, we determined the distribution of the non-conserved pTRRs reported for the ENCODE intervals [26] and demonstrated the concordance between our data and the ENCODE-derived data.

\section{Results \\ Conserved and non-conserved PHOX2B non-coding elements direct expression}

We recently demonstrated that both conserved and nonconserved sequences tiled across the zebrafish phox $2 b$ locus frequently displayed regulatory control consistent with endogenous phox $2 b$ expression [29]. We, and others, have pointed out that the increased divergence among teleosts, compared with divergence among mammals, increases the likelihood that zebrafish functional sequence modules will have acquired additional substitutions, reducing overt conservation. Additionally, despite the duplicate presence of approximately $30 \%$ of genes in zebrafish, its genome is significantly more compact than mammalian genomes ([37,38]; 1.9 gigabase pairs in zebrafish versus 3.1 gigabase pairs in human genome). Consequently functional sequences therein likely exist in 
proportionally reduced sequence space. Taken together, these observations suggest that one is more likely to find functional non-conserved regulatory elements in teleosts than in mammals. However, it remains untested, until now, whether these observations can be wholly accounted for by the above explanations or whether they, in part, are representative of a broader trend in vertebrate genomes. We set out to determine whether the human $P H O X 2 B$ locus contains non-conserved functional sequences like its zebrafish ortholog. In our recent study of phox $2 b$, the non-coding sequence intervals proximal to the phox $2 b$ exons revealed more non-conserved regulatory sequences than those at distance of $10 \mathrm{~kb}$ or greater. Thus we postulated that, if they exist, human non-conserved PHOX2B regulatory elements would be identified most readily in the gene proximal region. We initially generated 11 Tol2based transgenic constructs tiling across all non-coding sequences contained within the PHOX2B introns (two intronic constructs) as well as those contained within an interval of $10 \mathrm{~kb} 5^{\prime}$ to the transcriptional start site (TSS) (nine 5' constructs). Synteny between mammalian and teleost PHOX2b/phox $2 b$ orthologs begins to break down 3' to the coding exons; thus we focused on intronic sequences and sequences 5 ' to the gene. We introduced each construct into $\geq 200$ G0 zebrafish embryos as described previously $[19,39]$ and assayed their ability to drive enhanced GFP (eGFP) reporter expression in cell populations consistent with phox $2 b$ during development. Although we, and others, have previously demonstrated that G0 analyses can provide a robust assay of regulatory elements when requiring concordant expression among many independent embryos $[15,19,39,40]$, we also recognize that such analyses can over look some signals that become obvious only upon transmission through the germ line. In these analyses we sought to limit the potential for such events by requiring consistent signal among $\geq 10 \%$ of injected embryos; these embryos were used to score for function. Only elements that drove reproducible tissue specific expression were considered regulatory elements; images of representative embryos are provided for each identified regulatory element (Figure 1; Figure 2). Although the selected G0 images demonstrate that identified regulatory elements directed expression in cell types overlapping the endogenous phox $2 b$ expression domains we cannot definitively attribute the extent of their regulatory control nor exclude the potential for regulatory control beyond those domains. We can, however, identify that they display concordant functions as regulatory elements.

In our recent analysis of the zebrafish phox $2 b$ locus, we demonstrated that among the many commonly used sequence conservation algorithms, phastCons performed with the highest sensitivity and specificity $[29,41]$. Thus in our comparisons of the functionality of conserved versus non-conserved sequences, we classified amplicons based on overlap with phastCons Placental Mammal Conserved Elements, 28-way Multiz Alignment intervals [41-43]. The amplicons generated in this experiment comprised a tiling path of nine non-intronic elements (seven conserved, two non-conserved), encompassing 10,244 bp of the total 10,562 bp in this region, and 1,721 bp of sequence contained within its two introns (one conserved, one nonconserved; Figure 1A; Additional file 1 Table S1).

As expected, seven of the eight human conserved sequences (HCS), within $10 \mathrm{~kb}$ of $\mathrm{PHOX} 2 \mathrm{~B}$, directed tissue-specific expression consistent with that of endogenous phox $2 b$, including expression in neuronal populations of the ventral diencephalon (VDi), cranial ganglia (CG), and hindbrain ( $\mathrm{Hb}$ )(Figure 1B). Additionally, two out of three assayed human non-conserved sequences (HNCS) in this interval also drove phox $2 b$ appropriate expression, consistent with observations in zebrafish. PHOX2B-HNCS-4.2 directed expression in the ventral diencephalon and the hindbrain, while $P H O X 2 B$ HNCS-5.3 directed expression in the forebrain and hindbrain (Figure $1 \mathrm{~B}$ ). By contrast $\mathrm{PHOX} 2 \mathrm{~B}-\mathrm{HNCS}+1.7$ failed to direct detectable reporter expression during the developmental time points analyzed (Table 1). Importantly, these data are consistent with our previous findings at the orthologous phox $2 b$ zebrafish locus [29] and are consistent with our underlying hypothesis.

\section{Orthologous PHOX2B/phox2b amplicons display overlapping functions}

Since $P H O X 2 B$ and its zebrafish ortholog are critical for neuronal development in both mammals and teleosts $[33,44]$, we posited that the corresponding human and zebrafish orthologous non-coding sequences in this interval would share largely overlapping functions. The assayed human tiling path amplicons included three elements (PHOX2B-HCS+0.6, PHOX2B-HCS-0.0, PHOX2BHCS-2.9) conserved to zebrafish; their orthologous conserved sequences (ZCS) are phox $2 b$-ZCS +0.5 , phox $2 b$ ZCS+0.0 and phox $2 b$-ZCS-1.4 (Table 2). PHOX2B-HCS0.0 directed expression in the ventral diencephalon and hindbrain in G0 fish (Figure 1B), overlapping with the hindbrain expression observed in the previously published G1 results using the orthologous zebrafish sequence (Table 2 and Additional file 1 Table S1; [29]). PHOX2B-HCS-2.9 directed reporter expression in the forebrain and hindbrain, overlapping the hindbrain reporter expression directed by its zebrafish ortholog (phox $2 b$-ZCS$1.4 ;$ [29]). Furthermore, although PHOX2B-HCS+0.6 drove reporter expression in the forebrain and cranial ganglia consistent with phox $2 b$, these sites also extend beyond the observed regulatory control of the orthologous zebrafish phox $2 b$-ZCS+0.5 sequence. The incomplete nature of overlap in regulatory control observed for these 
A.

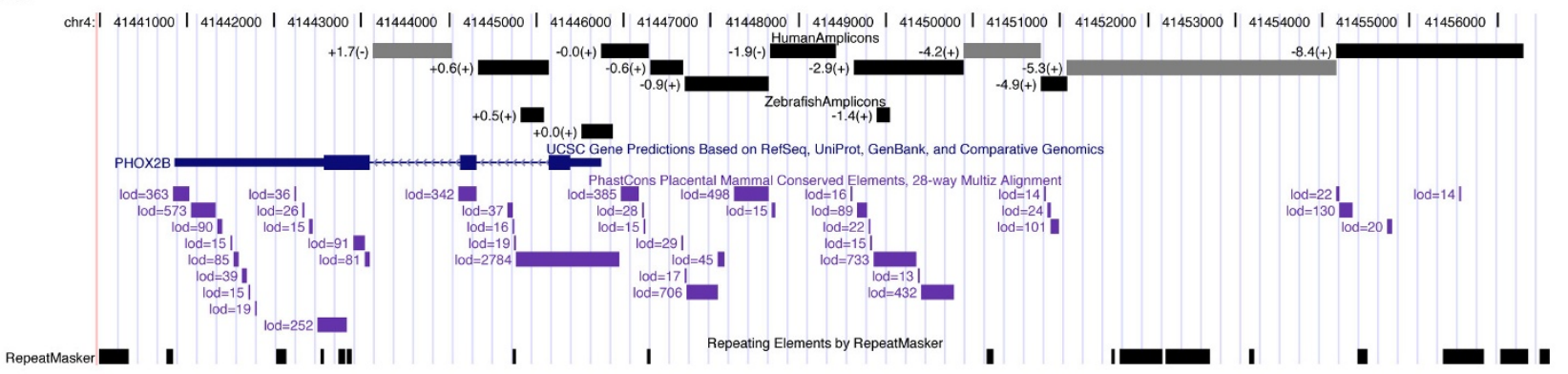

B.
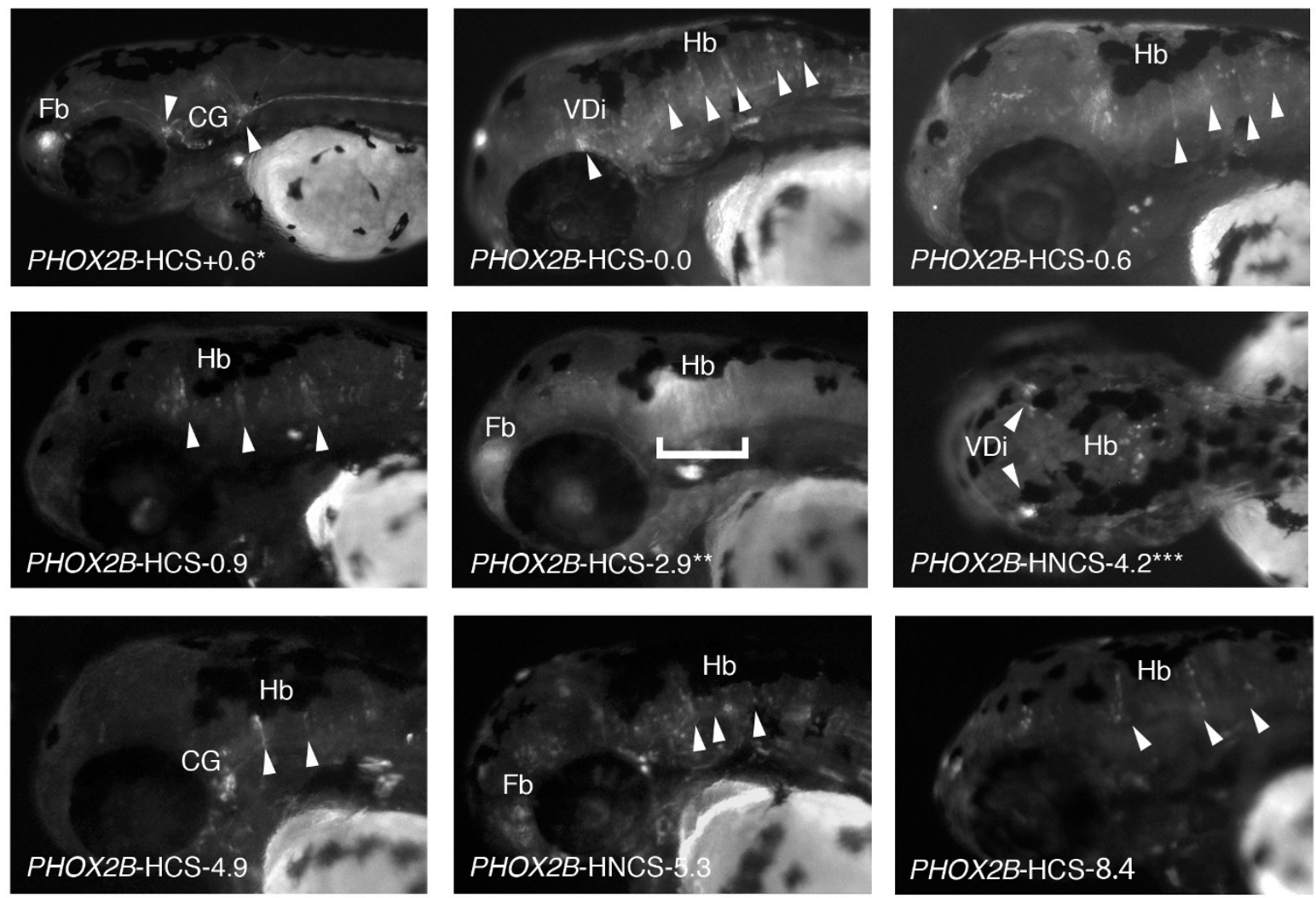

\section{Figure I}

Conserved and non-conserved amplicons tiled across the $P H O X 2 B$ proximal region direct $P H O X 2 B$ appropriate expression. (a) The human PHOX2B promoter proximal region (chr4:4I,440,000-4I,456,600; hg I8) was divided into II amplicons (total size II,965 base pairs) excluding exons, 5' UTR, and 3' UTR, according to whether intervals contained PhastCons Placental Mammal Conserved Elements, 28-way Multiz Alignment sequences [4I]. The amplicons are represented as gray scale rectangles: black (PHOX2B-HCS); gray (PHOX2B-HNCS); black (zebrafish alignment). Amplicon names are defined by their distance from the $P H O X 2 B$ transcriptional start site and are displayed as custom tracks on the UCSC Genome browser http://genome.ucsc.edu[54] (b) Lateral images of G0 48-hpf zebrafish embryos exhibiting PHOX2B appropriate expression with element name marked on picture. Fb, Forebrain; VDi, Ventral Diencephalon; Hb, Hindbrain; CG, Cranial Ganglia; SC, Spinal Cord; ENS, Enteric Nervous System. *GI embryo at 72-hpf. **GI embryo at 48-hpf. *** Dorsal photo. 

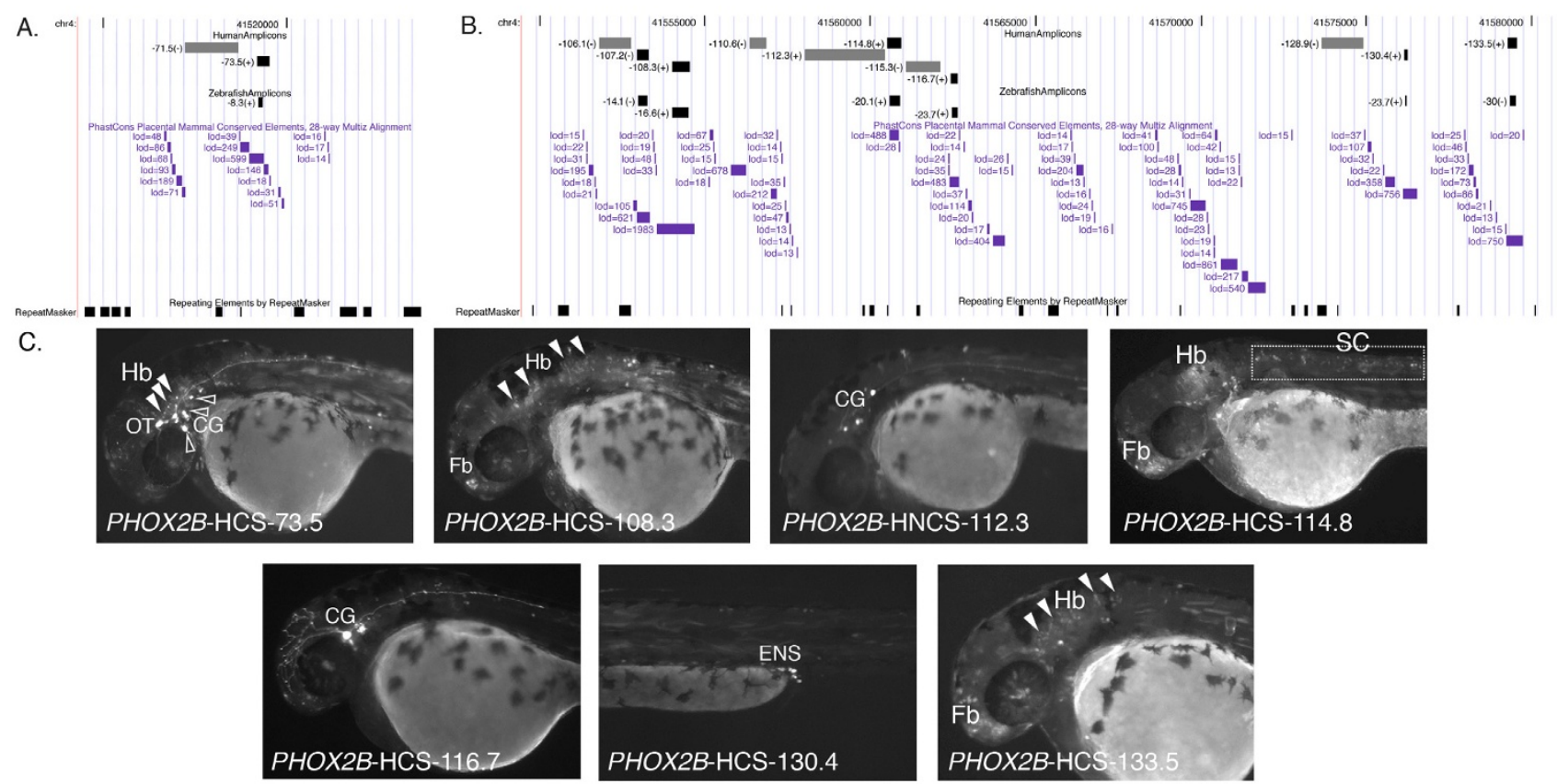

\section{Figure 2}

PHOX2B human distal conserved sequences demonstrate activity consistent with orthologous zebrafish sequences. The interval displayed as a custom track on UCSC Genome browser [54]. The amplicons are represented as gray scale rectangles: black (conserved), gray (non-conserved), black (zebrafish alignment). (A) Region containing region aligning to phox2b-ZCS -8.3 (chr4:4I,5I6,36I-4I,52I,080; hg I8) (B) Region containing aligning to phox2b-ZCS - I6.6,phox2b-ZCS-20.l, phox2b-ZCS -23.7 and phox2b-ZCS-30.0. (chr4:4I,549,434-4I,580, I42; hg I8) (C) Lateral images of G0 transgenic zebrafish embryos corresponding to functional human conserved (PHOX2B-HCS -73.5, PHOX2B-HCS - I08.3, PHOX2B-HCS-II4.8, PHOX2B-HCS - I 16.7, PHOX2B-HCS - I30.4 and PHOX2B-HCS-I33.5), and human non-conserved (PHOX2B-HNCS - I I2.3) amplicons. Fb, Forebrain; OT, Oculomotor and Trochlear Motor Progenitors; Hb, Hindbrain; CG, Cranial Ganglia; SC, Spinal Cord; ENS, Enteric Nervous System. Closed arrow-heads point to hindbrain expression. Open arrow-heads point to cranial ganglia expression.

elements may reflect lineage-specific adaptation of a common ancestral functional element (Figure 1B, Table 1, Table 2). Importantly, analyses of PHOX2B-HCS+0.6 and PHOX2B-HCS +2.9 in G0 were validated by passage through the germ line and analysis of $=2$ independent lines per construct. The resulting observations (Figure 1B) were consistent between G0 and G1 embryos analyzed.

To further test our hypothesis, we expanded our analyses of human $P H O X 2 B$ sequences orthologous to the remain- ing four previously identified phox $2 b$ zebrafish enhancers (phox $2 b$-ZCS -8.3, phox $2 b$-ZCS-16.6, phox $2 b$-ZCS-20.1 and phox $2 b$-ZCS-23.7) that align to the human PHOX2B locus [29]. These sequences were scattered over an interval in excess of $130 \mathrm{~kb} 5^{\prime}$ to the PHOX2B gene. Zebrafish sequence elements phox $2 b$-ZCS- 8.3 , phox $2 b$-ZCS- 16.6 and phox $2 b$-ZCS-20.1 aligned with the human sequences PHOX2B-HCS-73.5, PHOX2B-HCS-108.3 and PHOX2BHCS-114.8, respectively (Figure 2A, 2B; Table 2; Additional file 1 Table S1). However, the zebrafish amplicon

Table I: Functional non-conserved elements exhibit non-uniform distribution.

\begin{tabular}{lll}
\hline Functional non-conserved sequences & Zebrafish phox $2 b$ & Human PHOX2B \\
\hline$\leq 10 \mathrm{~kb}$ from gene & $3 / 5$ functional & $2 / 3$ functional \\
$>10 \mathrm{~kb}$ from gene & $1 / 8$ functional & $1 / 6$ functional
\end{tabular}

The distribution and function of tested non-conserved elements at the zebrafish phox $2 b$ locus [29] and human $P H O X 2 B$ locus are detailed; elements are grouped according to position (less than or greater than $10 \mathrm{~kb}$ of the $\mathrm{PHOX} 2 \mathrm{~B}$ gene region). 
Table 2: Human PHOX2B elements conserved to zebrafish phox2b locus demonstrate activity consistent with orthologous zebrafish sequences.

\begin{tabular}{|c|c|c|c|c|}
\hline Human Amplicon & $\begin{array}{l}\text { Expression } \\
\text { (HCS) }\end{array}$ & Zebrafish Amplicon & $\begin{array}{l}\text { Expression } \\
\text { (ZCS) }\end{array}$ & Coincident Control \\
\hline PHOX2B-HCS+0.6* & $\mathrm{Fb}, \mathrm{CG}$ & phox $2 b-Z C S+0.5$ & $\mathrm{Hb}, \mathrm{SC}$ & No \\
\hline PHOX2B-HCS -0.0 & $\mathrm{VDi}, \mathrm{Hb}$ & phox $2 b-Z C S+0.0$ & $\mathrm{Hb}, \mathrm{SC}$ & Yes \\
\hline PHOX2B-HCS -2.9* & $\mathrm{Fb}, \mathrm{Hb}$ & phox $2 b-Z C S-I .4$ & $\mathrm{Mb}, \mathrm{Hb}, \mathrm{SC}$ & Yes \\
\hline PHOX2B-HCS -73.5 & OT, CG, Hb & phox $2 b-Z C S-8.3$ & $\mathrm{Mb}, \mathrm{Hb}, \mathrm{CG}$ & Yes \\
\hline PHOX2B-HCS -108.3 & $\mathrm{Fb}, \mathrm{Hb}$ & phox $2 b$-ZCS - 16.6 & $\mathrm{Mb}, \mathrm{Hb}$ & Yes \\
\hline PHOX2B-HCS-II4.8 & $\mathrm{Fb}, \mathrm{Hb}, \mathrm{SC}$ & phox2b-ZCS-20.I & $\mathrm{Hb}$ & Yes \\
\hline PHOX2B-HCS - II6.7 & CG & phox2b-ZCS -23.7 & $\mathrm{Hb}, \mathrm{SC}$, ENS & No \\
\hline PHOX2B-HCS - 130.4 & ENS & phox2b-ZCS -23.7 & $\mathrm{Hb}, \mathrm{SC}$, ENS & Yes \\
\hline
\end{tabular}

HCS expression is pattern driven by human amplicon in G0 zebrafish embryos (* indicates GI expression pattern). ZCS expression is pattern driven by orthologous zebrafish amplicon in GI zebrafish embryos [29]. Overlap is categorized as Yes, tissue overlap in expression patterns, but additional tissues seen; No, no overlap in expression. Fb, Forebrain; OT, Oculomotor and trochlear motor Progenitors; VDi, Ventral diencephalon; $\mathrm{Hb}$, Hindbrain; CG, Cranial ganglia,; SC, Spinal cord ENS, Enteric nervous system.

phox2b-ZCS-23.7, which represents a tight cluster of highly conserved sequence intervals $(4.2 \mathrm{~kb})$ aligned to human genomic sequences scattered over $13.8 \mathrm{~kb}$, more than $115 \mathrm{~kb} 5^{\prime}$ to the human PHOX2B TSS. We selected intervals PHOX2B-HCS-116.7 and PHOX2B-HCS-130.4, aligning to phox $2 b$-ZCS-23.7, as representative sequences for evaluation (Figure 2B, Table 2 and Additional file 1 Table S1).

PHOX2B-HCS-73.5 directed reporter expression in the oculomotor and trochlear motor progenitors, cranial ganglia and hindbrain, consistent with the cranial ganglia and hindbrain reporter expression pattern exhibited by the zebrafish orthologous element phox2b-ZCS-8.3 (Figure 2A, 2C) [29]. However, in contrast with its zebrafish ortholog, PHOX2B-HCS-73.5 did not direct detectable expression in the midbrain. As noted above, this may reflect lineage-specific adaptation of the common ancestral regulatory sequences by the zebrafish and human phox $2 b /$ PHOX $2 B$ loci. It may also reflect, in part, the mosaic nature of the reporter expression observed in G0 embryos. However, one might reasonably expect that cell populations present in lower abundance within the embryo might be more likely to be overlooked in mosaics and not vice versa. Amplicon PHOX2B-HCS-108.3 directed reporter expression in the forebrain and hindbrain, overlapping the hindbrain expression its orthologous zebrafish sequence directed (phox2b-ZCS-16.6; Figure 2C, Table 2 and Additional file 1 Table S1, [29]). Moving more distal from the PHOX2B coding sequence, PHOX2B-HCS-114.8 directed expression in the forebrain, hindbrain, and spinal cord, once again overlapping the tissue specific hindbrain regulatory activity of its zebrafish orthologous sequence (phox2b-ZCS-20.1; Figure 2C; Table 2; [29]). Interestingly, although PHOX2B-HCS-116.7 was functionally active within the central nervous system, its tissue-specific regulatory control was discrete from that of phox 2 b-ZCS-23.7, to which it aligns. PHOX2B-HCS-116.7 directed expression within cranial ganglia, contrasting with the hindbrain, spinal cord and enteric nervous system expression demonstrated by the orthologous zebrafish sequence phox2b-ZCS-23.7 (Figure 2C, Table 2; [29]). Despite lying immediately proximal to an uncharacterized primate-specific predicted gene, $\mathrm{PHOX} 2 \mathrm{~B}$-HCS130.4 directed enteric nervous system-specific reporter expression overlapping the expression pattern displayed by phox2b-ZCS-23.7 (Figure 2C, Table 2 and Additional file 1 Table S1, [29]). These data are largely consistent with our underlying hypothesis that orthologous sequences drive overlapping expression patterns. Overall, six of the eight enhancer sequences that aligned between zebrafish and human overlapped in their regulatory control; these observations are, of course, consistent with their conservation and with their potentially important role in vertebrate nervous system development.

We then tested three human regions at the PHOX2B locus (PHOX2B-HCS-34.8, PHOX2B-HCS-107.2 and PHOX2BHCS-133.5) that aligned to conserved zebrafish phox $2 b$ sequences lacking detectable enhancer function (phox $2 b$ ZCS-3.5, phox2b-ZCS-14.1, and phox2b-ZCS-30.0, respectively) in our previous analysis (Figure 2A, 2B, [29]). Consistent with their zebrafish orthologs, neither PHOX2BHCS-34.8 nor PHOX2B-HCS-107.2 exhibited detectable regulatory control. PHOX2B-HCS-133.5, however, did display regulatory control, directing reporter expression in the forebrain and hindbrain (Figure 2C). Once again this observation may represent lineage-specific adaptation of an ancestral regulatory element; we also cannot exclude the possibility that the zebrafish element (phox $2 b$-ZCS30.0) may function at times outside the window examined (24-96 hour post fertilization). Consistent with our initial postulate, 8 of 11 assayed human sequences displayed regulatory control overlapping their zebrafish orthologs. Perhaps unsurprisingly, 8 of 11 human sequences conserved to zebrafish also displayed some 
spatial control within the nervous system that was absent from their zebrafish orthologs, consistent with lineagespecific variation in regulatory control.

\section{in vivo functional validation of skewed non-conserved regulatory element distribution at phox2b/PHOX2B}

We posited that non-conserved regulatory elements occur less frequently with an increasing distance from the gene. Further evaluation of our recent analysis at the zebrafish phox $2 b$ locus [29] suggests that three of five zebrafish nonconserved (ZNCS) regulatory elements within $10 \mathrm{~kb}$ of the gene exhibit function. Zebrafish elements phox $2 b$ ZNCS+6.7, phox $2 b-\mathrm{ZNCS}+5.6$ and phox $2 b-\mathrm{ZNCS}+3.1$ drove phox $2 b$ consistent expression, while phox $2 b$-ZNCS4.9 and phox $2 b$-ZNCS-5.9 did not exhibit regulatory activity [29]. However, only one of eight non-conserved zebrafish amplicons greater than $10 \mathrm{~kb}$ from the gene exhibited regulatory activity (phox $2 b$-ZNCS-27.9; Table 1; [29]). We posited that the human PHOX2B locus might exhibit the same characteristic distribution of non-conserved regulatory elements (Table 1).

To directly address this idea, we assayed six additional non-conserved sequences flanking functional distal regulatory elements conserved from zebrafish to human (Figure 2A-C, Additional file 1 Table S1). The non-conserved element PHOX2B-HNCS-71.5, which is adjacent to PHOX2B-HCS-73.5 (Figure 2a), exhibited no function upon injection of $\geq 200$ G0 zebrafish embryos. Upon injection and assay of comparable numbers of embryos with constructs PHOX2B-HNCS-106.1, PHOX2B-HNCS110.6, and PHOX2B-HNCS-112.3 (Figure 2B), only PHOX2B-HNCS-112.3 drove expression. This element directed reporter expression in the cranial ganglia, consistent with endogenous phox $2 b$ expression (Figure 2C). Additionally, we selected and analyzed elements PHOX2B-HNCS-115.3 and PHOX2B-HNCS-128.9, immediately flanking the conserved enhancers $P H O X 2 B$ HCS-116.7, PHOX2B-HCS-130.4, respectively; both nonconserved amplicons failed to direct reproducible reporter expression in any tissues at all time points examined (Figure $2 \mathrm{~B}$ ). Thus, of these six human distal non-conserved sequences tested, only PHOX2B-HNCS-112.3 displayed evidence of tissue-specific regulatory control (Table 1; Figure $2 \mathrm{~A}-\mathrm{C})$. These data are consistent with our earlier observations at the zebrafish phox $2 b$ locus (Table $1 ;$ [29]). If one examines the corresponding human and zebrafish data sets for sequences within $10 \mathrm{~kb}$ of the gene, they similarly suggest a bias in the distribution of functional nonconserved, non-coding sequences proximal to the gene. Comparison of the data generated at the human and zebrafish $P H O X 2 B /$ phox $2 b$ orthologs demonstrate that two of the three non-conserved elements tested in the PHOX2B (TSS-proximal) tiling path drove expression consistent with endogenous phox $2 b$ expression (Figure 1;
[29]), which agrees with the data generated at phox $2 b$ in zebrafish, for the same interval (Table 1; [29]).

While it remains possible that the observed skewed distribution of non-conserved, functional non-coding sequences is unique to $\mathrm{PHOX} 2 \mathrm{~B} / \mathrm{phox} 2 \mathrm{~b}$ or represents an artifact of a small sample size, we posited that it might also represent a more general characteristic of vertebrate genomes. When combining the zebrafish and human data, five of eight non-conserved elements within $10 \mathrm{~kb}$ of the gene exhibit regulatory function, while only two of 14 non-conserved gene distal elements exhibit regulatory function (Table 1; Figure 1; Figure 2; [29]). Taken together these data suggest that the non-conserved elements proximal to genes may have an increased probability of functioning as a transcriptional regulatory element. These in vivo observations at the $P H O X 2 B$ locus indicate a potential trend that functional non-conserved regulatory sequences may not be uniformly distributed with respect to genes; functional non-conserved amplicons display a slightly skewed distribution, with a higher frequency more proximal to genes and a lower frequency in more distal regions. We sought to test the generality of these observations to other loci using the recently published ENCODE data [25].

\section{The non-uniform distribution of non-conserved regulatory sequences represents a genome-wide phenomenon}

Until the completion of the human, mouse and other vertebrate genome sequences, the 5 ' putative promoter regions of genes were the primary site of inquiry for vertebrate regulatory sequences. Although existing functional data sets of regulatory sequences may consequently be enriched for these sequence intervals, the recently completed ENCODE project also reports a similar trend $[25,26,35]$. Prompted by the observation of a potential unequal distribution of conserved regulatory elements at the PHOX2B locus, we set out to determine whether the distribution of non-conserved regulatory elements also contributes to this genome wide trend. King and colleagues [26] recently identified 1,394 putative transcriptional regulatory regions (pTRRs) within the $1 \%$ of the human genome evaluated by ENCODE [25]. We examined this data, classifying pTRRs as conserved or non-conserved based upon whether they overlapped with PhastCons Placental Mammal Conserved Elements, 28way Multiz Alignment intervals [10,41,43]. We then applied the ENCODE defined sub-region identifiers (coding sequence, 5' UTR, 3' UTR, intronic proximal, intronic distal, intergenic proximal, or intergenic distal) to the pTRRs (Additional file 1 Table S2, Table 3). As with our analysis of non-conserved sequences at the PHOX2B and phox $2 b$ loci, we focused on pTRRs within intergenic proximal (intervals closer than $5 \mathrm{~kb}$ to nearest exon) and intergenic distal intervals (farther than $5 \mathrm{~kb}$ from the nearest 
Table 3: Distribution of putative transcriptional regulatory regions (pTRRs) identified by King et al. [26].

\begin{tabular}{|c|c|c|c|c|}
\hline Type of gene & $\begin{array}{l}\text { ENCODE Sub-regions } \\
\text { Analyzed }\end{array}$ & $\begin{array}{r}\text { Conserved PTRRs in sub- } \\
\text { region }\end{array}$ & $\begin{array}{r}\text { Non-conserved PTRRs in sub- } \\
\text { region }\end{array}$ & Base pairs in sub-region \\
\hline All & 5' UTR & 71 & 46 & 99,440 \\
\hline All & 3' UTR & 15 & 12 & 382,329 \\
\hline All & Intergenic Proximal & 61 & 163 & $2,429,196$ \\
\hline All & Intergenic Distal & 48 & 171 & $11,055,834$ \\
\hline All & Intronic Proximal & 173 & 457 & $8,903,959$ \\
\hline All & Intronic Distal & 55 & 122 & $6,462,925$ \\
\hline All & Coding sequences & 0 & 0 & 671,166 \\
\hline Developmental & Intergenic Proximal & 20 & 22 & 392,692 \\
\hline Developmental & Intergenic Distal & 5 & 20 & $1,636,075$ \\
\hline Non-developmental & Intergenic Proximal & 24 & 86 & 733,487 \\
\hline Non-developmental & Intergenic Distal & 10 & 51 & $2,309,353$ \\
\hline Non-gene Desert & Intergenic Distal & 39 & 159 & $7, \mid 47,316$ \\
\hline Gene Desert & Intergenic Distal & 9 & 12 & $3,908,518$ \\
\hline Gene Desert & Intergenic Proximal & 0 & 3 & 75,000 \\
\hline Non-gene Desert & Intergenic proximal & 61 & 160 & $2,354,196$ \\
\hline
\end{tabular}

PTRRs partitioned to ENCODE defined regions and grouped as conserved versus non-conserved based on pTRR overlap with PhastCons Placental Mammal Conserved Elements, 28-way Multiz Alignment [43]. Gene type of all represents analysis of whole ENCODE region. Developmental genes represent regions flanking genes labeled with Gene Ontology term GO:0032502, while non-developmental genes were those that were not labeled with GO:0032502. Gene deserts were ENCODE intervals overlapping regions $\geq 500$ kb without a Reference Sequence gene. Non-gene deserts were all sub-regions that did not overlap gene deserts. The "Base pairs in sub-region" column represents the sum of the genomic intervals represented by each type of sub-regions.

exon). After calculating the total number of conserved and non-conserved base pairs in each sub-region (see Methods for details on calculations), we then determined the density of non-conserved pTRRs within intergenic proximal and intergenic distal sub-regions (Table 4, Additional file
1, Table S2). Non-conserved pTRR density was defined as the number of non-conserved pTRRs in the region per non-conserved base pair in the region. Interestingly, the density of non-conserved pTRRs within intergenic proximal sub-regions was 4.33 fold higher than the density

Table 4: Non-conserved pTRR density is higher in intergenic proximal regions than intergenic distal regions.

\begin{tabular}{|c|c|c|c|c|c|}
\hline \multirow[b]{2}{*}{ Gene Type } & \multirow[b]{2}{*}{ ENCODE Sub-region } & \multicolumn{4}{|c|}{ PTRR Density } \\
\hline & & $\begin{array}{r}\text { Conserved pTRRs/ } \\
\text { Conserved bp }\end{array}$ & $\begin{array}{r}\text { Non-conserved } \\
\text { PTRRs/Non-conserved } \\
\text { bp }\end{array}$ & $\begin{array}{r}\text { Conserved pTRRS/ } \\
\text { Conserved Non-repeat } \\
\text { bp }\end{array}$ & $\begin{array}{r}\text { Non-conserved pTRRs } \\
\text { Non-conserved Non- } \\
\text { repeat bp }\end{array}$ \\
\hline All & 5' UTR & $1 / 457$ & $1 / 1,456$ & $1 / 410$ & $|/ 93|$ \\
\hline All & 3' UTR & $1 / 5,625$ & $1 / 24,829$ & $1 / 5,460$ & $1 / 18,610$ \\
\hline All & Intergenic Proximal & $1 / 1,185$ & $1 / 14,460$ & $\mathrm{I} / \mathrm{I}, 094$ & $1 / 7,005$ \\
\hline All & Intergenic Distal & $1 / 7,528$ & $|/ 62,54|$ & $\mathrm{I} / 7,073$ & $1 / 29,635$ \\
\hline All & Intronic Proximal & $1 / 1,463$ & $1 / 18,930$ & $1 / 1,356$ & $1 / 10,801$ \\
\hline All & Intronic Distal & $1 / 4,259$ & $\mathrm{I} / 5 \mathrm{I}, 055$ & $1 / 4,026$ & $|/ 28,23|$ \\
\hline Developmental & Intergenic Proximal & $|/|, 59 \mid$ & $1 / 16,403$ & $\mathrm{I} / \mathrm{I}, 508$ & $1 / 8,796$ \\
\hline Developmental & Intergenic Distal & $1 / 11,370$ & $|/ 78,96|$ & $1 / 10,7 \mid 4$ & $1 / 47,792$ \\
\hline Non-developmental & Intergenic Proximal & $1 / 867$ & $\mathrm{I} / 8,287$ & $|/ 82|$ & $1 / 3,619$ \\
\hline Non-developmental & Intergenic Distal & $1 / 6,160$ & $\mathrm{I} / 44,074$ & $1 / 5,776$ & $1 / 18,446$ \\
\hline Non-gene Desert & Intergenic Proximal & $\mathrm{I} / \mathrm{I}, 078$ & $1 / 14,303$ & $1 / 99 \mid$ & $1 / 6,877$ \\
\hline Gene Desert & Intergenic Proximal & $\mathrm{N} / \mathrm{A}$ & $1 / 22,824$ & $\mathrm{~N} / \mathrm{A}$ & $1 / 13,830$ \\
\hline Non-gene Desert & Intergenic Distal & $\mathrm{I} / 5,307$ & $1 / 43,650$ & $1 / 4,954$ & $1 / 19,047$ \\
\hline Gene Desert & Intergenic Distal & $1 / 17,151$ & $|/ 3| 2,847$ & $1 / 16,254$ & $1 / 151,566$ \\
\hline
\end{tabular}

Density of putative transcriptional regulatory regions (PTRRS) identified by King et al. [26]. The ENCODE interval was partitioned into sub-regions. Gene type of all represents analysis of whole ENCODE region. Developmental genes represent regions flanking genes labeled with Gene Ontology term GO:0032502, while non-developmental genes were those that were not labeled with GO:0032502. Gene deserts were ENCODE intervals overlapping $\geq 500 \mathrm{~kb}$ regions without a Reference Sequence gene. Non-gene deserts were all sub-regions that did not overlap gene deserts. Density of PTRRs was calculated by dividing the total number of conserved or non-conserved base pairs in the ENCODE defined region by number of conserved or non-conserved PTRRs in the ENCODE defined region. N/A= not applicable due to lack of conserved pTRRs in gene desert intergenic proximal regions 
within intergenic distal sub-regions (Table 4, Table 5). Although potentially interesting, we also noted that these observations could be significantly confounded by several factors; we thus addressed each in turn.

First, much of the genome is composed of repeats whose distribution is non-uniform, we thus set out to determine if the difference in non-conserved pTRR density between intergenic proximal and intergenic distal regions was due to density of repetitive DNA elements within evaluated intervals. We then calculated the total number of conserved and non-conserved repeat base pairs in each subregions, which where then used to calculate the number of conserved non-repeat base pairs and non-conserved nonrepeat base pairs in each sub-region (Additional file 1, Table S2, See methods for details of calculations). Adjusted for repeats, the density of non-conserved pTRRs is 4.23 times higher in intergenic proximal regions than in intergenic distal sub-regions, suggesting that the trend observed of higher non-conserved pTRR density proximal to the gene compared to distal to the gene is not due to a difference in repetitive element density in the sub-regions (Table 4; Table 5; Additional file 1 Table S2).

Second, developmental genes are reported to require more regulatory control modules than non-developmental genes [45-47]. We therefore asked whether the density of pTRRs differed between sequences flanking developmental and non-developmental genes. We utilized Gene Ontology (GO) to differentiate between developmental and non-developmental genes, using GO term GO:0032502 to define developmental genes [48]. Using "GO Slimmer" http://amigo.geneontology.org/cgi-bin/ amigo/slimmer; [49]), 78 unique genes from the ENCODE intervals labeled with GO:0032502 were identified as developmental genes, while 152 genes from the
ENCODE region were correspondingly identified as nondevelopmental. The fold change in densities of non-conserved pTRRs from intergenic proximal to intergenic distal for developmental genes (4.81 fold change in density) and non-developmental (5.32 fold change in density) were consistent with those in analysis of all ENCODE intergenic proximal and intergenic distal sub-regions (4.32 fold change in density; Table 4; Table 5). Additionally, the fold change in density between the intergenic proximal and intergenic distal sub-regions when adjusting for repeats remained similar for both the developmental genes ( 5.43 fold change in density) and non-developmental genes (5.10 fold change in density; Additional file 1, Table S3; Table 4; Table 5).

Finally, we sought to determine whether our observations still held true in comparisons of gene desert intervals alone and those specifically excluding gene deserts. To examine the effect of gene deserts on pTRR density, we compared gene desert intergenic proximal and intergenic distal sub-regions to non-gene desert intergenic proximal and intergenic distal regions. Intergenic distal sub-regions were manually curated to identify the sub-regions that overlapped gene deserts, defined as intervals $\geq 500 \mathrm{~kb}$ without a National Center for Biotechnology Information Reference Sequence gene [50,51]. After analysis was performed as described above on the gene desert and nongene desert sub-regions (Additional file 1, Table S4), the pTRR densities were once again calculated (Table 4). The gene desert intergenic distal non-conserved pTRR density was nearly an order of magnitude ( 7.17 fold) lower ( 1 non-conserved PTRR per 312,847 non-conserved base pairs) than that of non-gene desert intergenic distal regions (1 non-conserved pTRR per 43,650 base pairs), consistent with our underlying hypothesis that as distance from the gene increases, the density of non-conserved reg-

Table 5: Fold change in PTRR density of intergenic versus intergenic distal regions.

\begin{tabular}{|c|c|c|c|c|}
\hline \multirow[b]{2}{*}{ Gene Type } & \multicolumn{4}{|c|}{ Fold change of PTRR density between Intergenic Proximal and Intergenic Distal Sub-regions } \\
\hline & $\begin{array}{r}\text { Conserved pTRRs/ } \\
\text { Conserved bp }\end{array}$ & $\begin{array}{l}\text { Non-conserved pTRRs/ } \\
\text { Non-conserved bp }\end{array}$ & $\begin{array}{r}\text { Conserved pTRRs/Conserved } \\
\text { Non-repeat bp }\end{array}$ & $\begin{array}{r}\text { Non-conserved pTRRs/ } \\
\text { Non-conserved } \\
\text { Non-repeat bp }\end{array}$ \\
\hline All & 6.35 & 4.33 & 6.47 & 4.23 \\
\hline Developmental & 7.15 & 4.81 & 7.10 & 5.43 \\
\hline Non-developmental & 7.10 & 5.32 & 7.04 & 5.10 \\
\hline Gene Desert & N/A & $|3.7|$ & $\mathrm{N} / \mathrm{A}$ & 10.96 \\
\hline Non-gene Desert & 4.92 & 3.05 & 5.00 & 2.77 \\
\hline
\end{tabular}

Gene type of all represents analysis of whole ENCODE region. Developmental genes represent regions flanking genes labeled with Gene Ontology term GO:0032502, while non-developmental genes were those that were not labeled with GO:0032502. Gene deserts were ENCODE intervals overlapping $\geq 500 \mathrm{~kb}$ regions without a reference sequence gene. Non-gene deserts were all sub-regions that did not overlap gene deserts. Fold change in gene density was calculated for each gene type by dividing the intergenic proximal PTRR density by the intergenic distal PTRR density (Table 4). The pTRR densities were calculated for conserved pTRRs divided by conserved bp, non-conserved pTRRs divided by non-conserved bp, conserved pTRRs divided by conserved non-repeat bp and non-conserved pTRRs divided by non-conserved non-repeat bp $(T a b l e ~ 3)$. N/A $=$ not applicable due to lack of conserved pTRRs in gene desert intergenic proximal regions. 
ulatory elements decreases. However, since gene desert flanking intergenic proximal regions represent such a small fraction $(0.25 \%)$ of the total base pairs analyzed (Additional file 1 table S4), it is difficult to draw any conclusions on PTRR density for this subset alone. However, we can say that when comparing non-gene desert intergenic proximal regions to non-gene desert intergenic distal regions, we still observe a 3.02 fold change in nonconserved pTRR density. These data indicate that while gene deserts may contribute to the skewed PTRR density, there remains a significant fraction that cannot be accounted for by their effect.

\section{Discussion}

The identification of functional non-coding regulatory sequence relies heavily upon commonly applied metrics of constraint. However, our previous analysis at the zebrafish phox $2 b$ locus identified numerous functional non-coding regulatory sequences that were not under any detectable constraint [29], raising into question the frequency with which current approaches overlook functional elements. However, the large evolutionary distance between teleosts reduces the ability to detect constraint, so we undertook a similar analysis using the human PHOX2B locus.

The human PHOX2B and zebrafish phox $2 b$ gene proximal regions exhibited similar densities of both conserved and non-conserved functional elements, suggesting that our original observations at the zebrafish phox $2 b$ locus may not simply be an artifact of the increased evolutionary distance among teleosts compared with mammals. Furthermore, the numbers of non-conserved functional regulatory sequences decreased with increasing distance from the gene for both the zebrafish and human phox $2 b /$ $P H O X 2 B$ loci. We and others have proposed that the existence of non-conserved regulatory elements may result from lineage specific regulatory elements, transcription factor binding site shuffling, or elements falling below the threshold of detectable conservation $[23,24,26,29]$. Consistent with these postulates, analyses among more closely related species may increase the sensitivity and decrease the specificity with which one identifies true functional elements based upon conservation alone.

While previous studies have commented on the enrichment of regulatory elements proximal to genes $[25,26,35]$, the relative distribution of non-conserved regulatory elements had not been assessed in detail. We directly addressed this question in vivo and, using the ENCODE data set, in silico. Our analysis of ENCODEdefined pTRRs $[25,26]$ is consistent with the in vivo data both presented here for human $P H O X 2 B$ and published previously for the zebrafish phox $2 b$ ortholog [29], as well as with previously published predictions from cell line derived data [27]. We demonstrated that ENCODE-identified pTRRs [26] lacking conserved sequence intervals defined by phastCONS were present at a 4.33 fold higher density in intergenic proximal regions compared to the intergenic distal region (Table 4, Table 5). These trends were maintained even when accounting for repeat density or class-specific biases associated with developmental versus non-developmental genes. Also consistent with our underlying hypothesis, we noted a lower density of intergenic distal pTRRs in gene desert regions than in non-gene desert regions. This observation is also consistent with data generated by Roh et al. [27], who reported a similar enrichment of proximal non-conserved putative enhancers identified by histone acetylation patterns in vitro. Collectively these data suggest that the fraction of regulatory elements that can be detected by conservation alone may be smaller than previously believed.

\section{Conclusion}

The human and zebrafish $P H O X 2 B / p h o x 2 b$ data taken in combination with previously published data [27] and our analysis of the publicly available ENCODE data $[25,26]$ suggests that although conservation is a robust strategy to find functional sequences, implementation of this strategy alone will potentially overlook significant numbers of functional elements, particularly in regions proximal to genes. Importantly, these findings may significantly impact the search for regulatory variation underlying disease risk. The data suggest that although sequence conservation is a valid and often informative starting point for the identification of biologically functional sequences, there are frequently functional sequences that lie beneath that predictive radar. These observations suggest that the risk of overlooking non-conserved regulatory sequences at this level of constraint decreases with increasing distance from a gene.

\section{Methods \\ Selection and amplification of human non-coding sequences}

The sequences studied were in the region corresponding to chr4:41,443,127-41,579,542 in the human March 2006 (hg18) build. Using standard PCR conditions, sequences (Additional file 1 Table S1) were amplified off of human genomic DNA and separately subcloned into the pT2GWcfosEGFP vector, a Tol2-based transgenic reporter construct $[19,29,39]$. We, and others, have previously shown this to be a reliable screen for enhancer activity $[15,19,39]$. 333 base pairs were omitted from the tiling path due to primer design issues; the non-conserved amplicons were designed to have $0 \%$ overlap with the elements identified within the PhastCons Placental Mammal Conserved Elements, 28-way Multiz Alignment track [43]. 


\section{Fish care}

All zebrafish were raised, bred, and staged according to standard protocols at $28^{\circ} \mathrm{C}[52,53]$ and under protocols approved by the Johns Hopkins University Animal Care and Use Committee.

\section{Embryo injections and analysis}

Putative regulatory elements subcloned into the pT2GWcfosEGFP reporter construct were injected into wild-type G0 AB zebrafish embryos [39]. Reporter expression directed by each construct was then evaluated in $\geq 200$ live G0 mosaic embryos at 24, 48, 72 and 96 hours post fertilization, requiring that consistent signal is observed among $\geq 10 \%$ of injected embryos. Analysis of embryos was conducted using a Carl Zeiss Lumar V12 Stereo-microscope with AxioVision version 4.5 software.

\section{Analysis of ENCODE data \\ Defining ENCODE regions for analysis}

The human genome sequence (hg17) regions studied were obtained using the UCSC Genome Browser http:// genome.ucsc.edu[54] based on ENCODE coordinates [25]. The corresponding 44 regions encompass 29,998,060 base pairs. From the Galaxy2ENCODE (GENCODE) data sets available on the Galaxy database, partitioned intervals representing the ENCODE region were obtained as a custom track ([55,56]; http:// main.g2.bx.psu.edu/). The ENCODE sequence data was partitioned into 7 sub-regions: coding sequence; 5' UTR; 3 ' UTR; intronic proximal (intronic $\leq 5 \mathrm{~kb}$ from an exon); intronic distal (intronic $>5 \mathrm{~kb}$ from an exon); intergenic proximal, between genes $\leq 5 \mathrm{~kb}$ from an exon; and intergenic distal, between genes $>5 \mathrm{~kb}$ from an exon [55]. The partitioned ENCODE region was composed of 10,689 regions representing 30,717,051 base pairs, 718,991 base pairs more than the region represented by UCSC Genome Browser defined ENCODE regions. Using the Galaxy Database Subtraction tool, we determined that 167,090 of the extra base pairs were accounted for by partitioned subregions that lay outside the UCSC genome browser defined regions, which were excluded from the analysis. Using the Galaxy Database Base Coverage tool, we determined that the rest of the remaining 551,901 excess base pairs arose from redundant partitioning of the same genomic intervals into multiple sub-regions. Manual curation of the region was used to remove the redundancy, leaving only 6,789 base pairs of overlap $(0.02 \%$ of the ENCODE data set). The curated ENCODE partition sub-regions consisted of 10,052 intervals equaling $30,004,849$ base pairs. The Galaxy Database Base Coverage tool was used to confirm that the manually curated ENCODE partitioned sub-regions had the same actual base coverage as the UCSC defined ENCODE regions (29,998,060 base pairs). To confirm the accuracy of our partitions the total base pairs in each sub-regions were summed, and were shown to be equal to the total base pairs in the manually curated ENCODE partitioned subregions.

\section{Defining data sets for analysis}

Using the UCSC Table Browser, the human genomic coordinates (hg18) for the PhastCons Placental Mammal Conserved Elements, 28-way Multiz Alignment, which are not available in hg17, overlapping the ENCODE region were downloaded as a custom track. The Galaxy Database Liftover Convert Genome Coordinates tool was used to convert the PhastCons Placental Mammal Conserved Elements, 28-way Multiz Alignment ([41-43]), genomic intervals from hg18 to hg17. These elements were then partitioned into ENCODE sub-regions using the Galaxy Database Gencode Partition tool. The total PhastCons Placental Mammal Conserved Elements, 28-way Multiz Alignment base pairs in each sub-region were then summed and termed conserved base pairs. The total nonconserved base pairs were then calculated by subtracting the conserved base pairs from each sub-region from the total base pairs for each sub-region (Additional file 1, Table S2).

To examine the effects of repeats on pTRR density, we calculated the amount of repeat DNA in each ENCODE subregion. The hg17 repeat masker track for the UCSC defined ENCODE regions was downloaded in a custom track. To remove any overlap from repeat elements, the Galaxy Database Merge tool was used to concatenate overlapping repeat regions into single intervals. The merged repeats were then partitioned into ENCODE sub-regions using the Galaxy Database Gencode Partition tool. The total repeat base pairs were calculated for each sub-region using the merged partitioned repeats. To confirm that no repeats were lost in the Merge program, the Galaxy Database Base Coverage tool was used to confirm that the repeat masker and merged repeat intervals covered the same total genomic region (Additional file 1 Table S2).

To calculate the number of repeats that overlap with conserved regions, the Galaxy Database Intersection tool was used to calculate the intervals of base pair overlap between the PhastCons Placental Mammal Conserved Elements, 28-way Multiz Alignment intervals and merged repeat masker intervals. The regions of these overlaps were termed conserved repeats. The conserved repeats were then partitioned into ENCODE sub-regions using the Galaxy Database Gencode Partition tool. The total numbers of base pairs for each sub-region were summed. The conserved repeat base pairs were used to calculate the number of non-conserved repeat base pairs by subtracting the total number of conserved repeat base pairs from the number of total repeat base pairs for each sub-region. To calculate the non-conserved non-repeat base pairs, the non-con- 
served repeat base pairs were subtracted from the total non-conserved base pairs for each sub-region. To calculate the conserved non-repeat base pairs, the total conserved repeat base pairs were subtracted from the total conserved base pairs for each sub-region (Additional file 1 Table S2).

\section{pTRR analysis}

The hg 17 genomic coordinates for putative transcriptional regulatory regions identified by the ENCODE project [26] were obtained at http://www.bx.psu.edu/ projects/encode pTRR. The UCSC Table Browser Intersection tool was then used to determine which pTRRs overlap the PhastCons Placental Mammal Conserved Elements, 28-way Multiz Alignment, termed conserved pTRRs, and those which do not, termed non-conserved pTRRs. The conserved and non-conserved pTRRs were then partitioned into the ENCODE sub-regions using the Galaxy Database Gencode Partition pool. The total number of conserved and non-conserved pTRRs for each sub-region was totaled (Table 3, Additional file 1 Table 2).

To calculate the density of pTRRs in each sub-region, the number of pTRRs in each sub-region was divided by the total number of base pairs in each sub-region, with a density of pTRRs per base pair. To calculate the density of conserved pTRRs in conserved regions, the total number of conserved pTRRs in each sub-region was divided by the total number of conserved base pairs in each sub-region. To calculate the density of non-conserved pTRRs, the number of non-conserved pTRRs in each sub-region was divided by the total number of non-conserved base pairs in each sub-region (Additional file 1 Table 2, Table 4).

To examine the effects of repeats on conserved pTRR density, the number of conserved pTRRs was divided by the number of conserved non-repeat base pairs in each subregion. Also, the number of non-conserved pTRRs was divided by the number of non-conserved non-repeat base pairs in each sub-region (Additional file 1 Table 2).

\section{Gene desert versus non-gene desert analysis}

To study the effects of gene deserts on pTRR density, the gene desert regions of the ENCODE region were identified by manually curating the intergenic distal sub-regions to identify regions that had a minimum of $50 \mathrm{~kb}$ of overlap with $\mathrm{a}=500 \mathrm{~kb}$ region that did not contain a National Center for Biotechnology Information Reference Sequence gene [50,51]. All intergenic distal gene desert regions were expanded 500 base pairs in the $3^{\prime}$ and $5^{\prime}$ direction; these expanded regions were then used on the UCSC Table Browser intersection tool to find all intergenic proximal regions flanking gene desert regions. The total number of base pairs, non-conserved base pairs, conserved base pairs, repeat base pairs, conserved repeat base pairs, non-conserved repeat base pairs, conserved non- repeat base pairs, non-conserved non-repeat base pairs, conserved pTRRs, and non-conserved pTRRs were calculated for both the gene desert intergenic distal and intergenic proximal regions as described above. The totals of the gene desert regions were then subtracted from intergenic proximal and intergenic distal all genes totals to calculate non-gene desert statistics. The density of conserved pTRRs, non-conserved pTRRs and the repeat adjusted density of conserved pTRRs and non-conserved pTRRs was then calculated as described above (Additional file 1 Table 4).

\section{Analyses of Developmental versus Non-developmental Gene Intervals}

To determine whether pTRR density differed between flanking developmental genes and non-developmental genes, Gene Ontology terms were used to classify the genes in the ENCODE region [48]. Using the UCSC Table Browser, the table for MGC genes in the ENCODE region was downloaded. Gene ontology term GO:0032502 was chosen as a reference to define developmental genes versus non-developmental genes. The gene names off the MGC gene table were then entered into the GO slimmer tool (http://amigo.geneontology.org/cgi-bin/amigo/slim mer; [49]), with the setting All species databases, All evidence codes, GO Slimmer term GO:0032502. Of the genes input into GO slimmer, 230 total unique genes were recognized by the database. Gene names not recognized by GO slimmer were excluded from the analysis. Of these, 78 , labeled developmental genes, were labeled with the GO term GO:0032502, while 152 genes, termed nondevelopmental genes, were not labeled with GO:0032502. Manual curation was then used to create separate UCSC Genome Browser custom tracks containing the gene regions of the non-developmental and developmental genes respectively. These gene region intervals for both non-developmental and developmental genes were then expanded $7.5 \mathrm{~kb}$ in both the 5 ' and 3 ' direction, to allow identification of intergenic proximal and intergenic distal regions flanking the developmental and nondevelopmental regions. Using the UCSC Table Browser intersection tool, the overlap was found between the expanded developmental gene intervals and the intergenic proximal and intergenic distal sub-regions. Using the same method the overlap between the non-developmental gene expanded intervals and the intergenic proximal and intergenic distal sub-regions was also identified. To identify sub-regions that were positioned between a developmental gene and a non-developmental gene, and thus represented in both data sets, the UCSC Table Browser intersection tool was used to identify regions of overlap between the developmental gene intergenic proximal sub-regions and the non-developmental intergenic proximal sub-regions; and the overlap between the developmental gene intergenic proximal sub-regions and the 
non-developmental intergenic proximal sub-regions. These regions of overlap were excluded from the analysis due to their presence in both data sets. Using the UCSC Table Browser intersection tool the regions of overlap were subtracted from developmental and non-developmental gene intergenic proximal and intergenic distal subregions to leave four non-overlapping data sets termed developmental gene intergenic proximal non-overlap regions, developmental gene intergenic distal non-overlap regions, non-developmental gene intergenic proximal non-overlap regions, and non-developmental gene intergenic distal non-overlap regions. For these four data sets total number of base pairs, non-conserved base pairs, conserved base pairs, repeat base pairs, conserved repeat base pairs, non-conserved repeat base pairs, conserved nonrepeat base pairs, non-conserved non-repeat base pairs, conserved pTRRs, and non-conserved pTRR and appropriate PTRR densities were calculated as above (Additional file 1 Table 5).

\section{Authors' contributions}

ASM conceived this study. DMM and ZES performed analysis and imaging of zebrafish embryos. DMM, RMV and JH performed injections of zebrafish. DMM and ZES performed amplification and cloning of sequences. ZES performed analysis of ENCODE data. DMM, ZES, and ASM drafted manuscripts with revisions from JH and RMV. All authors read and approved by the final manuscript.

\section{Additional material}

\section{Additional file 1 \\ Supplementary tables S1-S5 \\ Click here for file \\ [http://www.biomedcentral.com/content/supplementary/1471- 2164-10-8-S1.pdf]}

\section{Acknowledgements}

This study was supported by funds from the NIH (NIGMS) to A.S.M. D.M.M. and Z.E.S. were also supported by NIH pre-doctoral training grant 5T32GM078/4. We also thank Rachel Stine for critical reading of this manuscript, and Amy Dinitz for her technical assistance.

\section{References}

I. Emison ES, McCallion AS, Kashuk CS, Bush RT, Grice E, Lin S, Portnoy ME, Cutler DJ, Green ED, Chakravarti A: A common sex-dependent mutation in a RET enhancer underlies Hirschsprung disease risk. Nature 2005, 434(7035):857-863.

2. Lettice LA, Heaney SI, Purdie LA, Li L, de Beer P, Oostra BA, Goode $D$, Elgar G, Hill RE, de Graaff E: A long-range Shh enhancer regulates expression in the developing limb and fin and is associated with preaxial polydactyly. Human molecular genetics 2003 , I 2(14): I725-I735.

3. Loots GG, Kneissel M, Keller H, Baptist M, Chang J, Collette NM, Ovcharenko D, Plajzer-Frick I, Rubin EM: Genomic deletion of a long-range bone enhancer misregulates sclerostin in Van Buchem disease. Genome research 2005, I 5(7):928-935.
4. Davidson $\mathrm{EH}$, Erwin $\mathrm{DH}$ : Gene regulatory networks and the evolution of animal body plans. Science (New York, NY) 2006, 3 I I (5762):796-800.

5. Stathopoulos A, Levine M: Genomic regulatory networks and animal development. Developmental cell 2005, 9(4):449-462.

6. Cretekos CJ, Wang Y, Green ED, Martin JF, Rasweiler JJt, Behringer RR: Regulatory divergence modifies limb length between mammals. Genes \& development 2008, 22(2): |4|-|5|.

7. McGregor AP, Orgogozo V, Delon I, Zanet J, Srinivasan DG, Payre F, Stern DL: Morphological evolution through multiple cis-regulatory mutations at a single gene. Nature 2007, 448(7 I 53):587-590.

8. Shapiro MD, Marks ME, Peichel CL, Blackman BK, Nereng KS, Jonsson B, Schluter D, Kingsley DM: Genetic and developmental basis of evolutionary pelvic reduction in threespine sticklebacks. Nature 2004, 428(6984):717-723.

9. Aparicio S, Morrison A, Gould A, Gilthorpe J, Chaudhuri C, Rigby P, Krumlauf $R$, Brenner $S$ : Detecting conserved regulatory elements with the model genome of the Japanese puffer fish, Fugu rubripes. Proceedings of the National Academy of Sciences of the United States of America 1995, 92(5): I 684- I688.

10. Blanchette M, Bataille AR, Chen X, Poitras C, Laganiere J, Lefebvre C, Deblois G, Giguere V, Ferretti V, Bergeron D, et al.: Genome-wide computational prediction of transcriptional regulatory modules reveals new insights into human gene expression. Genome research 2006, I6(5):656-668.

II. Grice EA, Rochelle ES, Green ED, Chakravarti A, McCallion AS: Evaluation of the RET regulatory landscape reveals the biological relevance of a HSCR-implicated enhancer. Hum Mol Genet 2005, I 4(24):3837-3845.

12. Marshall H, Studer M, Popperl H, Aparicio S, Kuroiwa A, Brenner S, Krumlauf $R$ : A conserved retinoic acid response element required for early expression of the homeobox gene Hoxb-I. Nature 1994, 370(6490):567-57I.

13. Nobrega MA, Pennacchio LA: Comparative genomic analysis as a tool for biological discovery. The Journal of physiology 2004, 554(Pt I):31-39.

14. Pennacchio LA, Loots GG, Nobrega MA, Ovcharenko I: Predicting tissue-specific enhancers in the human genome. Genome research 2007, I7(2):20I-2II.

15. Woolfe A, Goodson M, Goode DK, Snell P, McEwen GK, Vavouri T, Smith SF, North P, Callaway H, Kelly K, et al.: Highly conserved non-coding sequences are associated with vertebrate development. PLoS biology 2005, 3(I):e7.

16. Bejerano G, Pheasant M, Makunin I, Stephen S, Kent WJ, Mattick JS, Haussler D: Ultraconserved elements in the human genome. Science 2004, 304(5675): I $321-1325$.

17. Pennacchio LA, Ahituv N, Moses AM, Prabhakar S, Nobrega MA Shoukry M, Minovitsky S, Dubchak I, Holt A, Lewis KD, et al.: In vivo enhancer analysis of human conserved non-coding sequences. Nature 2006, 444(7 I I 8):499-502.

18. Visel A, Prabhakar S, Akiyama JA, Shoukry M, Lewis KD, Holt A, Plajzer-Frick I, Afzal V, Rubin EM, Pennacchio LA: Ultraconservation identifies a small subset of extremely constrained developmental enhancers. Nature genetics 2008, 40(2): I58-I60.

19. Fisher S, Grice EA, Vinton RM, Bessling SL, McCallion AS: Conservation of RET regulatory function from human to zebrafish without sequence similarity. Science 2006, 3 I 2(577 I):276-279.

20. Frazer KA, Narla G, Zhang JL, Rubin EM: The apolipoprotein(a) gene is regulated by sex hormones and acute-phase inducers in YAC transgenic mice. Nature genetics 1995, 9(4):424-431.

21. Margulies EH, Cooper GM, Asimenos G, Thomas DJ, Dewey CN, Siepel A, Birney E, Keefe D, Schwartz AS, Hou M, et al.: Analyses of deep mammalian sequence alignments and constraint predictions for I\% of the human genome. Genome research 2007 , I 7(6):760-774.

22. Prabhakar S, Poulin F, Shoukry M, Afzal V, Rubin EM, Couronne O, Pennacchio LA: Close sequence comparisons are sufficient to identify human cis-regulatory elements. Genome research 2006, I 6(7):855-863.

23. Keightley PD, Lercher MJ, Eyre-Walker A: Evidence for widespread degradation of gene control regions in hominid genomes. PLoS biology 2005, 3(2):e42.

24. Sanges R, Kalmar E, Claudiani P, D'Amato M, Muller F, Stupka E: Shuffling of cis-regulatory elements is a pervasive feature of the vertebrate lineage. Genome biology 2006, 7(7):R56. 
25. Consortium EP, Birney E, Stamatoyannopoulos JA, Dutta A, Guigo R, Gingeras TR, Margulies EH, Weng Z, Snyder M, Dermitzakis ET, et al.: Identification and analysis of functional elements in $1 \%$ of the human genome by the ENCODE pilot project. Nature 2007, 447(7| 46):799-8।6.

26. King DC, Taylor J, Zhang Y, Cheng Y, Lawson HA, Martin J, Analysis EgTRaMS, Chiaromonte F, Miller W, Hardison RC: Finding cis-regulatory elements using comparative genomics: some lessons from ENCODE data. Genome research 2007, 17(6):775-786.

27. Roh TY, Wei G, Farrell CM, Zhao K: Genome-wide prediction of conserved and nonconserved enhancers by histone acetylation patterns. Genome research 2007, 17(I):74-8I.

28. Yaragatti $M$, Basilico $C$, Dailey L: Identification of active transcriptional regulatory modules by the functional assay of DNA from nucleosome-free regions. Genome research 2008.

29. McGaughey DM, Vinton RM, Huynh J, Al-Saif A, Beer MA, McCallion AS: Metrics of sequence constraint overlook regulatory sequences in an exhaustive analysis at phox 2b. Genome research 2008, I8(2):252-260.

30. Amiel J, Laudier B, Attie-Bitach T, Trang H, de Pontual L, Gener B, Trochet D, Etchevers H, Ray P, Simonneau M, et al.: Polyalanine expansion and frameshift mutations of the paired-like homeobox gene PHOX2B in congenital central hypoventilation syndrome. Nature genetics 2003, 33(4):459-46I.

31. Benailly HK, Lapierre JM, Laudier B, Amiel J, Attie T, De Blois MC, Vekemans M, Romana SP: PMX2B, a new candidate gene for Hirschsprung's disease. Clinical genetics 2003, 64(3):204-209.

32. Pattyn A, Morin X, Cremer H, Goridis C, Brunet JF: Expression and interactions of the two closely related homeobox genes Phox2a and Phox2b during neurogenesis. Development (Cambridge, England) 1997, I 24(20):4065-4075.

33. Pattyn A, Morin X, Cremer H, Goridis C, Brunet JF: The homeobox gene Phox $2 b$ is essential for the development of autonomic neural crest derivatives. Nature 1999, 399(6734):366-370.

34. Trochet D, Bourdeaut F, Janoueix-Lerosey I, Deville A, de Pontual L, Schleiermacher G, Coze C, Philip N, Frebourg T, Munnich A, et al.: Germline mutations of the paired-like homeobox 2B (PHOX2B) gene in neuroblastoma. American Journal of Human Genetics 2004, 74(4):76I-764.

35. Zhang ZD, Paccanaro A, Fu Y, Weissman S, Weng Z, Chang J, Snyder $M$, Gerstein MB: Statistical analysis of the genomic distribution and correlation of regulatory elements in the ENCODE regions. Genome research 2007, I7(6):787-797.

36. Giresi PG, Kim J, McDaniell RM, lyer VR, Lieb JD: FAIRE (Formaldehyde-Assisted Isolation of Regulatory Elements) isolates active regulatory elements from human chromatin. Genome Res 2007, 17(6):877-885.

37. Freeman JL, Adeniyi A, Banerjee R, Dallaire S, Maguire SF, Chi J, Ng BL, Zepeda C, Scott CE, Humphray S, et al: Definition of the zebrafish genome using flow cytometry and cytogenetic mapping. BMC genomics 2007, 8: 195 .

38. Danio rerio Sequencing Project [http://www.sanger.ac.uk/ Projects/D rerio/]

39. Fisher S, Grice EA, Vinton RM, Bessling SL, Urasaki A, Kawakami K McCallion AS: Evaluating the biological relevance of putative enhancers using Tol2 transposon-mediated transgenesis in zebrafish. Nature protocols 2006, I(3):|297-1305.

40. Paparidis Z, Abbasi AA, Malik S, Goode DK, Callaway H, Elgar G, deGraaff E, Lopez-Rios J, Zeller R, Grzeschik KH: Ultraconserved non-coding sequence element controls a subset of spatiotemporal GLI3 expression. Development, growth \& differentiation 2007, 49(6):543-553.

41. Siepel A, Bejerano G, Pedersen JS, Hinrichs AS, Hou M, Rosenbloom K, Clawson H, Spieth J, Hillier LW, Richards S, et al: Evolutionarily conserved elements in vertebrate, insect, worm, and yeast genomes. Genome research 2005, I 5(8): I034-1050.

42. Blanchette M, Kent WJ, Riemer C, Elnitski L, Smit AF, Roskin KM, Baertsch R, Rosenbloom K, Clawson H, Green ED, et al.: Aligning multiple genomic sequences with the threaded blockset aligner. Genome research 2004, 14(4):708-7I5.

43. Miller W, Rosenbloom K, Hardison RC, Hou M, Taylor J, Raney B, Burhans R, King DC, Baertsch R, Blankenberg D, et al:: 28-way vertebrate alignment and conservation track in the UCSC Genome Browser. Genome research 2007, I 7(12): 1797-I808.

44. Elworthy S, Pinto JP, Pettifer A, Cancela ML, Kelsh RN: Phox2b function in the enteric nervous system is conserved in zebrafish and is soxl0-dependent. Mech Dev 2005, I 22(5):659-669.

45. Bejerano G, Pheasant M, Makunin I, Stephen S, Kent WJ, Mattick JS, Haussler D: Ultraconserved elements in the human genome. Science 2004, 304(5675): | $32 \mid-1325$.

46. Boffelli D, Nobrega MA, Rubin EM: Comparative genomics at the vertebrate extremes. Nature reviewsGenetics 2004, 5(6):456-465.

47. Ovcharenko I, Loots GG, Nobrega MA, Hardison RC, Miller W, Stubbs $L$ : Evolution and functional classification of vertebrate gene deserts. Genome research 2005, I5(I): I37-145.

48. Ashburner M, Ball CA, Blake JA, Botstein D, Butler H, Cherry JM, Davis AP, Dolinski K, Dwight SS, Eppig JT, et al.: Gene ontology: tool for the unification of biology. The Gene Ontology Consortium. Nature genetics 2000, 25(I):25-29.

49. Day-Richter J, Harris MA, Haendel M, Gene Ontology OBOEWG, Lewis S: OBO-Edit - an ontology editor for biologists. Bioinformatics (Oxford, England) 2007, 23(16):21 98-2200.

50. Nobrega MA, Ovcharenko I, Afzal V, Rubin EM: Scanning human gene deserts for long-range enhancers. Science 2003, 302(5644): 413.

5I. Pruitt KD, Tatusova T, Maglott DR: NCBI reference sequences (RefSeq): a curated non-redundant sequence database of genomes, transcripts and proteins. Nucleic acids research 2007:D6I-65.

52. Kimmel CB, Ballard WW, Kimmel SR, Ullmann B, Schilling TF: Stages of embryonic development of the zebrafish. Dev Dyn 1995, 203(3):253-310.

53. Westerfield M: The Zebrafish Book: a Guide for the Laboratory Use of Zebrafish (Danio rerio). 4th edition. Eugene, Oregeon: University of Oregon Press; 2000.

54. Kent W], Sugnet CW, Furey TS, Roskin KM, Pringle TH, Zahler AM, Haussler D: The human genome browser at UCSC. Genome Res 2002, I 2(6):996-1006

55. Blankenberg D, Taylor J, Schenck I, He J, Zhang Y, Ghent M, Veeraraghavan N, Albert I, Miller W, Makova KD, et al.: A framework for collaborative analysis of ENCODE data: making large-scale analyses biologist-friendly. Genome research 2007 17(6):960-964.

56. Taylor J, Schenck I, Blankenberg D, Nekrutenko A: Using galaxy to perform large-scale interactive data analyses. Curr Protoc Bioinformatics 2007, 10:10.5. Unit 10.15
Publish with Bio Med Central and every scientist can read your work free of charge

"BioMed Central will be the most significant development for disseminating the results of biomedical research in our lifetime. "

Sir Paul Nurse, Cancer Research UK

Your research papers will be:

- available free of charge to the entire biomedical community

- peer reviewed and published immediately upon acceptance

- cited in PubMed and archived on PubMed Central

- yours - you keep the copyright
BioMedcentral 